\title{
A NONCOMMUTATIVE HALF-ANGLE FORMULA
}

\section{GeORGE WiLLis}

\begin{abstract}
The half-angle formulæ, familiar from trigonometry, can be used to compute the polar decomposition of the operator on $l^{2}(\mathbb{Z})$ of convolution by $\delta_{0}+\delta_{1}$. This calculation is extended here to a non-commutative setting by computing the polar decomposition of certain convolution operators on the spaces of square integrable functions of free groups.
\end{abstract}

\section{INTRODUCTION}

It is shown here how to compute the polar decomposition of certain operators in the reduced group $C^{*}$-algebra of a free group. When the free group has one generator, that is, when we are dealing with the group of integers, the polar decomposition may be computed, as explained in section 1, by taking the Fourier transform and appealing to elementary formulæ from trigonometry. These are often called the 'double-angle formulæ' but the direction of the calculation makes 'half-angle formulæ' more appropriate here.

Motivating this computation is the desire to gain insight into noncommutative mathematics, $[3,4]$. The group of integers and its dual, the circle, are elementary and basic examples which are the source of a great deal of intuition and imagery in mathematics. Our image of the circle comes from elementary geometry, as do the half-angle formulæ used to compute the polar decomposition in the one generator case. Now nonabelian free groups are fundamentally important also but we have no readily available conception of their duals which might be used to compute the polar decompositions. By computing the polar decomposition in the noncommutative setting in some other way it may be that something will be learned about the geometrical nature, if any, of the dual of a free group.

The calculation of the polar decomposition is given in section 3 . The answer is intriguing and does suggest that there may well be some insight or interpretation behind it. That insight does not seem to be provided by the method of calculation used here however. Some ideas for using the polar decompositions to study the topological nature of the dual of a free group are discussed at the end of the paper.

Received 22nd January, 2003

I am very grateful for the valuable assistance of May Nilsen, supported by a research infrastructure grant, with the preparation of this paper.

Copyright Clearance Centre, Inc. Serial-fee code: 0004-9727/04 \$A2.00+0.00. 


\section{ThE COMMUTATIVE HALF-ANGLE FORMULA}

The familiar half-angle formulæ for trigonometric functions are the real and imaginary parts of the single equation

$$
1+e^{i \theta}=2 \cos \left(\frac{\theta}{2}\right) e^{i(\theta / 2)}
$$

which is just the polar form of the complex number $1+e^{i \theta}$, if $-\pi<\theta \leqslant \pi$. When (1.1) is represented in the complex plane (see Figure 1) it implies also a case of the theorem from elementary geometry that the angle subtended by a chord at the circumference of a circle is half the angle subtended by the chord at the centre of the circle.

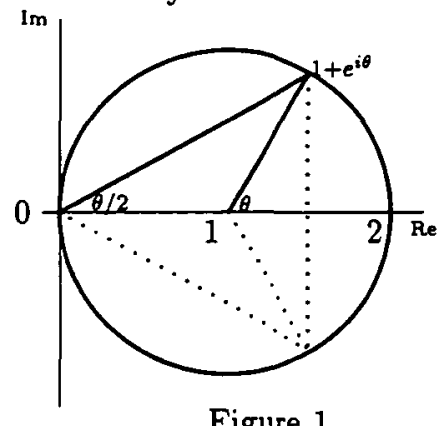

Figure 1.

Let $\mathbb{T}$ denote the circle group and parametrise $\mathbb{T}$ by $\theta$, where $-\pi<\theta \leqslant \pi$. Let $f$ be the continuous function on. $\mathbb{T}$ given by $f(\theta)=1+e^{i \theta}$. Then multiplication by $f$ defines a bounded operator $M_{f}$ of $L^{2}(\mathbb{T})$. Each operator on Hilbert space has a polar decomposition as the product of a positive operator and a partial isometry, $([7$, p. 68]). The formula (1.1) describes the polar decomposition of $M_{f}$ thus:

$$
M_{f}=M_{p} M_{u},
$$

where $M_{p}$ is the positive operator of multiplication by the continuous positive function $p(\theta)=2 \cos (\theta / 2)$, and $M_{u}$ is the unitary operator of multiplication by the discontinuous but measurable function $u(\theta)=e^{i(\theta / 2)}$.

Equations (1.1) and (1.2) are generalised in this paper in the form of their Fourier transforms. The Fourier transforms of the functions $f, p$ and $u$ in the previous paragraph are

$$
\begin{aligned}
& \widehat{f}(n)= \begin{cases}1, & \text { if } n \text { is } 0 \text { or } 1 \\
0, & \text { otherwise; }\end{cases} \\
& \widehat{p}(n)=\frac{(-1)^{n+1}}{\pi\left(n^{2}-(1 / 4)\right)} ; \quad \widehat{u}(n)=\frac{(-1)^{n+1}}{\pi(n-(1 / 2))} .
\end{aligned}
$$

The Fourier transform of $(1.1)$ is

$$
\widehat{f}=\widehat{u} * \widehat{p},
$$


where $*$ denotes the convolution product. Convolution by $\widehat{f}$ defines an operator $T$ on $\ell^{2}(\mathbb{Z})$ which is unitarily equivalent to $M_{f}$ under the Fourier transform. The operator $T$ belongs to the $C^{*}$-algebra $C_{r}^{*}(\mathbb{Z})$ of the regular representation of $\mathbb{Z}$ on $\ell^{2}(\mathbb{Z})$. The polar decomposition of $T$ is just the Fourier transform of (1.2), that is,

$$
T=U P,
$$

where $P$ is the positive operator of convolution by $\widehat{p}$ and $U$ is the unitary operator of convolution by $\widehat{u}$. Since $p$ is continuous, $P$ belongs to $C_{r}^{*}(\mathbb{Z})$. The operator $U$ does not belong to $C_{r}^{*}(\mathbb{Z})$ but does belong to the von Neumann algebra $V N(\mathbb{Z})$ of the regular representation of $\mathbb{Z}$. Not every operator in $C_{r}^{*}(\mathbb{Z})$ has an explicitly computable polar decomposition. It is the half-angle formulæ and Fourier transform which facilitate this computation of the polar decomposition of $T$.

\section{FREE GROUPS}

For each discrete group $G$, define the convolution of two functions in $\ell^{2}(G)$ by

$$
f \cdot * g(x)=\sum_{y \in G} f(y) g\left(y^{-1} x\right)
$$

where $x \in G$ and $f, g \in \ell^{2}(G)$. For each $x \in G, \delta_{x}$ will denote the function on $G$ defined by

$$
\delta_{x}(y)= \begin{cases}1, & \text { if } x=y, \\ 0, & \text { otherwise }\end{cases}
$$

Then the left regular representation of $G$ on $\ell^{2}(G)$ represents an element $x$ in $G$ by the unitary operator $\lambda(x): f \mapsto \delta_{x} * f\left(\left(f \in \ell^{2}(G)\right)\right.$. The $C^{*}$-algebra generated by $\{\lambda(x) \mid x \in G\}$ is the reduced $C^{*}$-algebra of $G$ and will be denoted by $C_{r}^{*}(G)$. The von Neumann algebra so generated will be denoted by $V N(G)$. If $S$ belongs to $V N(G)$, then $S$ is equal to the operator of convolution by the function $S \delta_{e}$, where $e$ denotes the identity element of $G$. Elements of $V N(G)$ and $C_{r}^{*}(G)$ may thus be represented by functions in $l^{2}(G)$.

Let $k$ be a positive integer and $\mathbb{F}_{k}$ be the free group with generators $x_{1}, x_{2}, \ldots x_{k}$. If $k=1$, then $\mathbb{F}_{k}$ is isomorphic to $\mathbb{Z}$ and we may suppose that $x_{1}$ is mapped by the isomorphism to 1 . In this case (1.4) describes the polar decomposition of the operator $\lambda(e)+\lambda\left(x_{1}\right)$. Now, for general $k$, let

$$
T:=\lambda(e)+\sum_{j=1}^{k} \lambda\left(x_{j}\right) .
$$

Then $T$ has polar decomposition

$$
T=U P
$$


where $P:=\left(T^{*} T\right)^{1 / 2}$ is a positive operator in $C^{*}\left(\mathbb{F}_{k}\right)$ and $U$ is a unitary operator in $V N\left(\mathbb{F}_{k}\right)$. The operator $U$ in the polar decomposition is generally a partial isometry only and is not unique. However in this case $T$ has zero kernel and dense range and it follows that $U$ is unitary and unique ([7]). Equation 2.1 is equivalent to

$$
\delta_{e}+\sum_{j=1}^{k} \delta_{x_{j}}=\widehat{u} * \widehat{p},
$$

where $p:=P \delta_{e}$ and $u:=U \delta_{e}$ belong to $\ell^{2}\left(\mathbb{F}_{k}\right)$.

The equation (2.2) and the computation of $p$ and $u$ will constitute the noncommutative half-angle formula. The justification for calling it a 'half-angle formula' is that the calculation proceeds by embedding $\mathbb{F}_{k}$ as a subgroup of index 2 in another group. There are several ways in which this can be done, see Section 4.2 below. In the case of $\mathbb{F}_{1}=\mathbb{Z}$, one such is the embedding $\mathbb{Z} \rightarrow(1 / 2) \mathbb{Z}$ which, from the point of view adopted here, is why the half angles appear in equation (1.1). However a different group must be used for the general case.

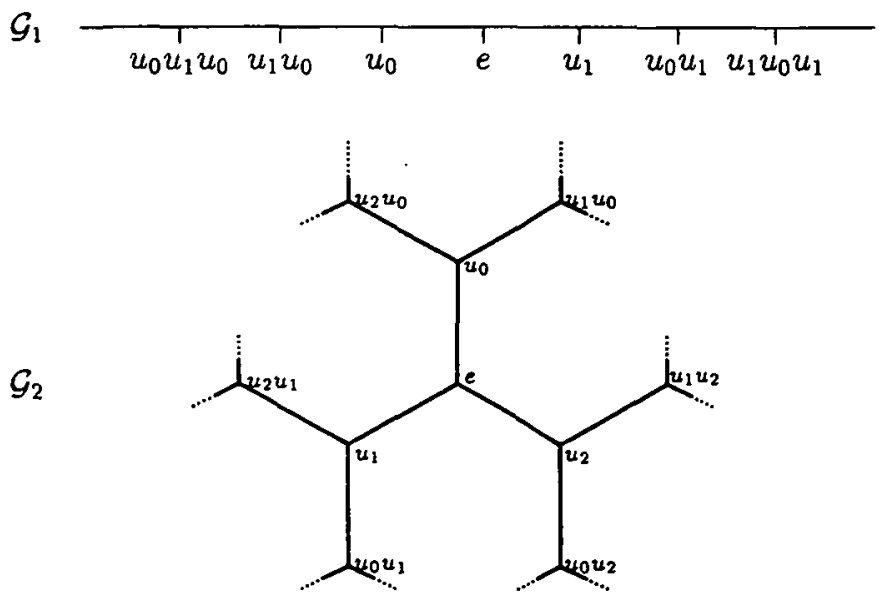

Figure 2.

Let $\mathbb{G}_{k}=\left\langle u_{0}, u_{1}, \ldots, u_{k} \mid u_{j}^{2}=e, j=0,1, \ldots, k\right\rangle$. It will be helpful to consider the Cayley graph of $\mathbb{G}_{k}$, which is the graph $\mathcal{G}_{k}$ whose vertices are the elements of $\mathbb{G}_{k}$ and where $x$ and $y$ are joined by an edge if $x=u_{j} y$ for some $j$. Since $\mathbb{G}_{k}$ is isomorphic to the free product of $k+1$ copies of $\mathbb{Z}_{2}=\mathbb{Z} / 2 \mathbb{Z}, \mathcal{G}_{k}$ is a homogeneous tree of degree $k+1$, see Figure 2. For each $j$ between 0 and $k$, the map $x \mapsto u_{j} x$ interchanges each vertex of $\mathcal{G}_{k}$ with one adjacent to it, while the map $x \mapsto x u_{j}$ is an automorphism of the tree 
which 'reflects' the graph about the midpoint of the edge joining $e$ and $u_{j}$. For each pair of elements $x$ and $y$ in $\mathbb{G}_{k}, d(x, y)$ will denote the distance between the corresponding vertices. Consider now the subgroup with index two in $\mathbb{G}_{k}$ consisting of those elements represented by words in $u_{0}, u_{1}, \ldots, u_{k}$ of even length. From the graphical point of view, this subgroup consists of those vertices of $\mathcal{G}_{k}$ which are an even distance from $e$. This subgroup is freely generated by the elements $u_{0} u_{j}, j=1,2, \ldots, u_{k}$ and we shall identify it with $\mathbb{F}_{k}$ by identifying $u_{0} u_{j}$ with the generator $x_{j}$ in $\mathbb{F}_{k}$ for each $j$. With $\mathbb{F}_{k}$ embedded in $\mathbb{G}_{k}$ in this way, we have

$$
\delta_{e}+\sum_{j=1}^{k} \delta_{x_{j}}=\delta_{u_{0}} *\left(\sum_{j=0}^{k} \delta_{u_{j}}\right) .
$$

This equation expresses the operator $\lambda(e)+\sum_{j=1}^{k} \lambda\left(x_{j}\right)$ as the product of the unitary operator $\lambda\left(u_{0}\right)$ and the operator $\sum_{j=0}^{k} \lambda\left(u_{j}\right)$ on $\ell^{2}\left(\mathbb{G}_{k}\right)$. We now find the polar decomposition of $\sum_{j=0}^{k} \lambda\left(u_{j}\right)$

\section{CoMPutation OF THE POLAR DECOMPOSITION}

Let $k$ be a positive integer and let $A:=\sum_{j=0}^{k} \lambda\left(u_{j}\right)$ in $C_{r}^{*}\left(\mathbb{G}_{k}\right)$. Then, since $u_{j}^{2}=e$ for each $j, A$ is self-adjoint and so the von Neumann algebra of operators on $\ell^{2}\left(\mathbb{G}_{k}\right)$ generated by $A$ is commutative. This von Neumann algebra will be denoted by $V N(A)$. The polar decomposition of $A$ is computed in the commutative algebra $V N(A)$ and for this it is necessary to describe some details of the structure of this von Neumann algebra. Calculations similar to those described here can be found in $[\mathbf{5}, \mathbf{2}, \mathbf{9}]$.

For each non-negative integer $m$, let $E_{m}:=\left\{x \in \mathbb{G}_{k} \mid d(e, x)=m\right\}$. Then $E_{0}=\{e\}$, $E_{1}=\left\{u_{0}, u_{1}, \ldots, u_{k}\right\}$ and, if $m>1,\left|E_{m}\right|=(k+1) k^{m-1}$. Let $A_{m}:=\sum_{x \in E_{m}} \lambda(x)$. Then $A_{0}$ is the identity operator, and $A_{1}=A$. Since

$$
A_{1} \lambda\left(u_{j}\right)=\lambda(e)+\sum_{i \neq j} \lambda\left(u_{i} u_{j}\right)
$$

summing over all $j$ yields

$$
A_{1}^{2}=(k+1) A_{0}+A_{2} .
$$

If $d(e, x)=m \geqslant 2$, then $x=u_{j} y$ for some $j$, where $d(e, y)=m-1$. Hence

$$
A_{1} \lambda\left(u_{j} y\right)=\lambda(y)+\sum_{i \neq j} \lambda\left(u_{i} u_{j} y\right)
$$

and summing over all $x=u_{j} y$ in $E_{m}$ gives

$$
A_{1} A_{m}=k A_{m-1}+A_{m+1}, \quad m=2,3,4^{\circ} \ldots
$$


It follows that each power of $A$ is a linear combination of the $A_{m}$ 's and hence that each operator, $S$, in $V N(A)$ may be expressed in the form

$$
S=\sum_{m=0}^{\infty} \alpha_{m} A_{m}
$$

or each $m$, the function $A_{m} \delta_{e}$ in $\ell^{2}\left(\mathbb{G}_{k}\right)$ is just the characteristic function of $E_{m}$, which we shall denote by $\chi_{m}$. Since these characteristic functions are mutually orthogonal, the coefficients in (3.2) may be found by

$$
\alpha_{m}=\frac{\left\langle S \delta_{e}, \chi_{m}\right\rangle}{\left\langle\chi_{m}, \chi_{m}\right\rangle}=\frac{1}{k^{m-1}(k+1)}\left\langle S \delta_{e}, \chi_{m}\right\rangle,
$$

where $\langle\cdot, \cdot\rangle$ denotes the inner product in $\ell^{2}\left(\mathbb{G}_{k}\right)$.

A function $f$ in $\ell^{2}\left(\mathbb{G}_{k}\right)$ is called radial if the value $f(x)$ depends only on the distance of $x$ from $e$. The characteristic functions $\chi_{m}$ are radial and so, if $S$ belongs to $V N(A)$, then $S \delta_{e}=\sum_{m=0}^{\infty} \alpha_{m} \chi_{m}$ is a radial function in $\ell^{2}\left(\mathbb{G}_{k}\right)$. Hence $V N(A)$ is an algebra of operators of convolution by radial functions on $\mathbb{G}_{k}$. The subspace of all radial functions in $\ell^{2}\left(\mathbb{G}_{k}\right)$ will be denoted by $\ell_{R}^{2}\left(\mathbb{G}_{k}\right)$. This subspace is invariant under $V N(A)$.

Since $A$ is self-adjoint, the $C^{*}$-algebra generated by $A$ is isomorphic via the Gelfand representation to the algebra of continuous functions on the spectrum of $A$. This spectrum is computed by Pytlik in [8] in the case when the homogeneous tree has even degree. Essentially the same calculation applies to homogeneous trees of odd degree to yield that the spectrum of $A$ is the interval $[-2 \sqrt{k}, 2 \sqrt{k}]$. (Note that the $k$ used here is 1 less than twice the value of ' $k$ ' in [8].) It follows from (3.1) and (3.2) that each of the operators $A_{m}$ is a polynomial in $A$ and so is mapped by the Gelfand representation to a polynomial function $\phi_{m}$ on $[-2 \sqrt{k}, 2 \sqrt{k}]$. From (3.2) we see that

$$
\phi_{0}(t)=1, \quad \phi_{1}(t)=t
$$

and for any integer $m \geqslant 2, \phi_{m}(t)$ satisfies the recurrence relation

$$
\phi_{m+1}(t)=t \phi_{m}(t)-k \phi_{m-1}(t)
$$

Note that $\phi_{m}$ is an even function when $m$ is even and an odd function when $m$ is odd.

The map $S \mapsto\left\langle S \delta_{e}, \delta_{e}\right\rangle$ is a state on the $C^{*}$-algebra generated by $A$ and thus corresponds to a probability measure $\mu$ on $[-2 \sqrt{k}, 2 \sqrt{k}]$ such that, for $\phi$ and $\psi$ in $C([-2 \sqrt{k}, 2 \sqrt{k}])$

$$
\left\langle\phi(A) \delta_{e}, \psi(A) \delta_{e}\right\rangle=\int_{-2 \sqrt{k}}^{2 \sqrt{k}} \phi(t) \bar{\psi}(t) d \mu(t) .
$$

Now $C([-2 \sqrt{k}, 2 \sqrt{k}])$ is dense in $L^{2}([-2 \sqrt{k}, 2 \sqrt{k}], \mu)$ and

$$
\left\{\phi(A) \delta_{e} \mid \phi \in C([-2 \sqrt{k}, 2 \sqrt{k}])\right\}
$$


is dense in $\ell_{R}^{2}\left(\mathbb{G}_{k}\right)$ because it contains $\operatorname{sp}\left\{A_{m} \delta_{e}\right\}$. Hence the map $\phi \mapsto \phi(A) \delta_{e}$ extends to a unitary map $\mathcal{U}$ from $L^{2}([-2 \sqrt{k}, 2 \sqrt{k}], \mu)$ onto $\ell_{R}^{2}\left(\mathbb{G}_{k}\right)$. This unitary defines an equivalence between $L^{\infty}([-2 \sqrt{k}, 2 \sqrt{k}], \mu)$ and $V N(A)$. Each $S$ in $V N(A)$ is equal to $\phi(A)$ for some $\phi$ in $L^{\infty}([-2 \sqrt{k}, 2 \sqrt{k}], \mu)$, where $\phi(A)=\mathcal{U} \circ M_{\phi} \circ \mathcal{U}^{-1}, M_{\phi}$ being the multiplication operator on $L^{2}([-2 \sqrt{k}, 2 \sqrt{k}], \mu)$ determined by $\phi$. It follows then from (3.4) and (3.6) that, if $S=\phi(A)$ belongs to $V N(A)$, then $S=\sum_{m=0}^{\infty} \alpha_{m} A_{m}$, where

$$
\alpha_{m}=\frac{1}{k^{m-1}(k+1)} \int_{-2 \sqrt{k}}^{2 \sqrt{k}} \phi(t) \phi_{m}(t) d \mu(t) .
$$

The measure $\mu$ is computed by Pytlik in [8, Theorem 5.1]. It is

$$
d \mu(t)=\frac{k+1}{2 \pi} \frac{\sqrt{4 k-t^{2}}}{(k+1)^{2}-t^{2}} d t
$$

Note that $\mu$ is an even measure.

The polar decomposition of $A$ can now be computed with the aid of the functional calculus in $V N(A)$. Thus

$$
A=V P,
$$

or $P=|A|\left(=\left(A^{2}\right)^{1 / 2}\right)$ and $V=\operatorname{sgn}(A)$, where

$$
\operatorname{sgn}(t)= \begin{cases}1, & \text { if } t>0 \\ 0, & \text { if } t=0 \\ -1, & \text { if } t<0\end{cases}
$$

By (3.7) and (3.8), $V=\sum_{m=0}^{\infty} \alpha_{m} A_{m}$, where

$$
\begin{aligned}
\alpha_{m} & =\frac{1}{k^{m-1}(k+1)} \int_{-2 \sqrt{k}}^{2 \sqrt{k}} \operatorname{sgn}(t) \phi_{m}(t) d \mu(t) \\
& = \begin{cases}0, & \text { if } m \text { is even } \\
\frac{1}{\pi k^{m-1}} \int_{0}^{2 \sqrt{k}} \phi_{m}(t) \frac{\sqrt{4 k-t^{2}}}{(k+1)^{2}-t^{2}} d t, & \text { if } m \text { is odd. }\end{cases}
\end{aligned}
$$

We now evaluate the integral in (3.11).

The functions $\phi_{m}$ satisfy the recurrence relation (3.5). It follows that for $m \geqslant 2$,

$$
\phi_{m+2}(t)=\left(t^{2}-2 k\right) \phi_{m}(t)-k^{2} \phi_{m-2}(t),
$$

thus for odd $m$, that is letting $m=2 n+1$,

$$
\phi_{2 n+3}(t)=\left(t^{2}-2 k\right) \phi_{2 n+1}(t)-k^{2} \phi_{2 n-1}(t),
$$


for $n \geqslant 1$. It can be shown by induction that, for $n \geqslant 0$,

$$
\phi_{2 n+1}(t)=-t \sum_{i=0}^{n}\left(t^{2}\right)^{i}\left\{\left(\begin{array}{c}
n+i+1 \\
)
\end{array}\right) n-i k+\left(\begin{array}{c}
n+i \\
n-i-1
\end{array}\right)\right\}(-k)^{n-i-1},
$$

where $\left(\begin{array}{l}a \\ 0\end{array}\right)=1$ and $\left(\begin{array}{c}a \\ -1\end{array}\right)=0$, for any integer $a$.

The change of variable $t=2 \sqrt{k} s$ in the integral (3.11) yields

$$
\alpha_{2 n+1}=\frac{4}{\pi k^{2 n-1}} \int_{0}^{1} \phi_{2 n+1}(2 \sqrt{k s}) \frac{\sqrt{1-s^{2}}}{(k+1)^{2}-4 k s^{2}} d s .
$$

Under this change of variable (3.12) and (3.13) become

$$
\begin{aligned}
& \phi_{2 n+3}(2 \sqrt{k} s)=\left(4 k s^{2}-2 k\right) \phi_{2 n+1}(2 \sqrt{k} s)-k^{2} \phi_{2 n-1}(2 \sqrt{k} s), \text { and } \\
& \phi_{2 n+1}(2 \sqrt{k} s)=-2 \sqrt{k} s \sum_{i=0}^{n}\left(4 k s^{2}\right)^{i}\left\{\left(\begin{array}{c}
n+i+1 \\
n-i
\end{array}\right) k+\left(\begin{array}{c}
n+i \\
n-i-1
\end{array}\right)\right\}(-k)^{n-i-1}
\end{aligned}
$$

Now define

$$
P_{n}(k, s):=-\sum_{i=0}^{n}\left(4 k s^{2}\right)^{i}\left\{\left(\begin{array}{c}
n+i+1 \\
n-i
\end{array}\right) k+\left(\begin{array}{c}
n+i \\
n-i-1
\end{array}\right)\right\}(-k)^{n-i-1}
$$

and note that $\phi_{2 n+1}(2 \sqrt{k} s)=2 \sqrt{k} s P_{n}(k, s)$. Then, by (3.14),

$$
P_{n+1}(k, s)=\left(4 k s^{2}-2 k\right) P_{n}(k, s)-k^{2} P_{n-1}(k, s) .
$$

Now we make a further change of variable, setting $s=\sqrt{1-y^{2}}$. Then

$$
\begin{aligned}
\alpha_{2 n+1} & =\frac{4}{\pi k^{2 n-1}} \int_{0}^{1} \frac{P_{n}(k, s)}{(k+1)^{2}-4 k s^{2}} \sqrt{1-s^{2}} 2 \sqrt{k} s d s \\
& =\frac{8 \sqrt{k}}{\pi k^{2 n-1}} \int_{0}^{1} \frac{P_{n}\left(k, \sqrt{1-y^{2}}\right) y^{2}}{(k-1)^{2}+4 k y^{2}} d y .
\end{aligned}
$$

An induction argument using (3.16) shows that $P_{n}\left(k, \sqrt{1-y^{2}}\right)=P_{n}(-k, y)$. Hence

$$
\alpha_{2 n+1}=\frac{8 \sqrt{k}}{\pi k^{2 n-1}} \int_{0}^{1} \frac{y^{2} P_{n}(-k, y)}{(k-1)^{2}+4 k y^{2}} d y .
$$

The next step in computing the integral (3.17) is polynomial long division, which may be carried out inductively using (3.16), to yield

$$
\frac{y^{2} P_{n}(-k, y)}{(k-1)^{2}+4 k y^{2}}=\frac{k^{n-1}}{4}\left(\sum_{i=0}^{n}\left(-4 y^{2}\right)^{i} \sum_{j=0}^{n-i}\left(\begin{array}{c}
n+i-j-1 \\
n-i-j
\end{array}\right) k^{j}-\frac{k^{n}(k-1)^{2}}{(k-1)^{2}+4 k y^{2}}\right)
$$


where we take $\left(\begin{array}{c}-1 \\ 0\end{array}\right)=1$ and $\left(\begin{array}{c}a-1 \\ a\end{array}\right)=0$ for all $a \geqslant 1$. We can now evaluate the integral (3.17):

$$
\begin{aligned}
\alpha_{2 n+1} & =\frac{2}{\pi}\left(\frac{\sqrt{k}}{k^{n}} \sum_{i=0}^{n} \sum_{j=0}^{n-i} \frac{(-4)^{i}}{2 i+1}\left(\begin{array}{c}
n+i-j-1 \\
n-i-j
\end{array}\right) k^{j}-(k-1) \arctan \frac{1}{\sqrt{k}}\right) \\
& =\frac{2}{\pi}\left(\frac{\sqrt{k}}{k^{n}} \sum_{i=0}^{n} \sum_{l=i}^{n} \frac{(-4)^{i}}{2 i+1}\left(\begin{array}{c}
l+i-1 \\
l-i
\end{array}\right) k^{n-l}-(k-1) \arctan \frac{1}{\sqrt{k}}\right), \\
& =\frac{2}{\pi}\left(\sqrt{k} \sum_{l=0}^{n}\left\{\sum_{i=0}^{l} \frac{(-4)^{i}}{2 i+1}\left(\begin{array}{c}
l+i-1 \\
l-i
\end{array}\right)\right\} k^{-l}-(k-1) \arctan \frac{1}{\sqrt{k}}\right) \\
& =\frac{2}{\pi}\left(\sqrt{k}+\sqrt{k} \sum_{l=1}^{n} \frac{(-1)^{l} 4 l}{(2 l-1)(2 l+1)} k^{-l}-(k-1) \arctan \frac{1}{\sqrt{k}}\right),
\end{aligned}
$$

where we have used the identity

$$
\sum_{i=0}^{l} \frac{(-4)^{i}}{2 i+1}\left(\begin{array}{c}
l+i-1 \\
l-i
\end{array}\right)=\frac{(-1)^{l} 4 l}{(2 l-1)(2 l+1)}
$$

for $l \geqslant 1$.

Note that the Laurent series expansion for $\left(\left(1 / x^{2}\right)-1\right) \arctan x$ yields

$$
(k-1) \arctan \frac{1}{\sqrt{k}}=\sqrt{k}+\sqrt{k} \sum_{l=1}^{\infty} \frac{(-1)^{l} 4 l}{(2 l-1)(2 l+1)} k^{-l},
$$

and so $\alpha_{2 n+1}=(2 / \pi) R_{2 n+1}(1 / \sqrt{k})$, where $R_{2 n+1}(x)$ is the remainder in the degree $2 n-1$ approximation to $\left(1 /\left(x^{2}\right)-1\right) \arctan x$. We see then that $\alpha_{2 n+1} \stackrel{n}{\rightarrow} 0$ as it ought.

Note also that

$$
R_{2 n+1}\left(\frac{1}{\sqrt{k}}\right)=(k-1) Q_{2 n+1}\left(\frac{1}{\sqrt{k}}\right)+\frac{(-1)^{n}}{2 n+1}\left(\frac{1}{\sqrt{k}}\right)^{2 n-1}
$$

where $Q_{2 n+1}(1 / \sqrt{k})$ is the remainder term in the Taylor series for $\arctan x$. Hence

$$
\alpha_{2 n+1}=\frac{2}{\pi}\left[(k-1) Q_{2 n+1}\left(\frac{1}{\sqrt{k}}\right)+\frac{(-1)^{n+1}}{2 n+1}\left(\frac{1}{\sqrt{k}}\right)^{2 n-1}\right] .
$$

Recalling (3.11), we have therefore that

$$
V=\sum_{m=0}^{\infty} \alpha_{m} A_{m}=\lambda(\widehat{v})
$$

where $\widehat{v}=\sum_{m=0}^{\infty} \alpha_{m} \chi_{m}=\sum_{n=0}^{\infty} \alpha_{2 n+1} \chi_{2 n+1}$. 
Since $V$ is an involution, see (3.10), the operator $P$ appearing in (3.9) satisfies

$$
\begin{aligned}
P=V A & =\lambda\left(\widehat{v} * \chi_{1}\right) \\
& =\alpha_{1}(k+1) \chi_{0}+\alpha_{1} \chi_{2}+\sum_{n=1}^{\infty} \alpha_{2 n+1}\left(k \chi_{2 n}+\chi_{2 n+2}\right), \quad \text { by }(3.2), \\
& =\alpha_{1}(k+1) \chi_{0}+\sum_{n=1}^{\infty}\left(\alpha_{2 n-1}+k \alpha_{2 n+1}\right) \chi_{2 n} .
\end{aligned}
$$

It may be checked that for $n \geqslant 1$,

$$
\begin{aligned}
\alpha_{2 n-1} & +k \alpha_{2 n+1} \\
= & \frac{2}{\pi}\left[(\sqrt{k})^{3}-\frac{1}{3} \sqrt{k}+\sqrt{k} \sum_{l=1}^{n-1} \frac{(-1)^{l} 4}{(2 l-1)(2 l+3)} k^{-l}-\left(k^{2}-1\right) \arctan \frac{1}{\sqrt{k}},\right] \\
= & \frac{2}{\pi} S_{2 n-1}\left(\frac{1}{\sqrt{k}}\right), .
\end{aligned}
$$

where $S_{2 n-1}(x)$ is the remainder in the degree $2 n-3$ approximation to $\left(\left(1 / x^{4}\right)-1\right) \arctan x$. Hence, putting

$$
\beta_{m}= \begin{cases}\frac{2}{\pi} \sqrt{k}(k+1), & \text { if } m=0 \\ \frac{2}{\pi} S_{2 n-1}\left(\frac{1}{\sqrt{k}}\right), & \text { if } m=2 n, n \geqslant 1 \\ 0, & \text { if } m=2 n-1\end{cases}
$$

and $\hat{p}=\sum_{m=0}^{\infty} \beta_{m} \chi_{m}$, we have $P=\lambda(\widehat{p})$. Note that $\widehat{p}$ is supported on words in $\mathbb{G}_{k}$ of even length and so $\widehat{p}$ is in fact a function on $\mathbb{F}_{k}$.

From (2.3) we have

$$
\begin{aligned}
\delta_{e}+\sum_{j=1}^{k} \delta_{x_{j}} & =\delta_{u_{0}} *\left(\sum_{j=0}^{k} \delta_{u_{j}}\right) \\
& =\delta_{u_{0}} *(\hat{v} * \widehat{p})=\widehat{u} * \widehat{p},
\end{aligned}
$$

where $\widehat{u}=\delta_{u_{0}} * \widehat{v}$. The operator $U=\lambda(\widehat{u})$ is unitary in $V N\left(\mathbb{F}_{k}\right)$ and $P=\lambda(\widehat{p})$ is positive in $C_{r}^{*}\left(\mathbb{F}_{k}\right)$ as required.

\section{REMARKS.}

4.1. Since $V$ is unitary, $\left\|\sum \alpha_{m} \chi_{m}\right\|_{2}=1$, that is

$$
\sum_{m=0}^{\infty}\left|\alpha_{m}\right|^{2}(k+1) k^{m-1}=1
$$


so that

$$
\begin{aligned}
\sum_{n=0}^{\infty}\left(R_{2 n+1}\left(\frac{1}{\sqrt{k}}\right)\right)^{2} k^{2 n} & =\sum_{n=0}^{\infty}\left((k-1) Q_{2 n+1}\left(\frac{1}{\sqrt{k}}\right)+\frac{(-1)^{n+1}}{2 n+1}\left(\frac{1}{\sqrt{k}}\right)^{2 n-1}\right)^{2} k^{2 n} \\
& =\frac{\pi^{2}}{4(k+1)} .
\end{aligned}
$$

When $k=1$ this reduces to the familiar identity $\sum_{m \text { odd }} 1 / m^{2}=\pi^{2} / 8$.

4.2. The embedding of $\mathbb{F}_{k}$ as an index two subgroup of $\mathbb{G}_{k}$ is only one of several embeddings which will produce the same result. If $k$ is odd, then $\mathbb{F}_{k}$ is isomorphic to the subgroup of $\mathbb{F}_{(1 / 2)(k+1)}$ consisting of words of even length. In particular, $\mathbb{F}_{1}$ is isomorphic to $\mathbb{Z}$, which may be embedded in the free product $\mathbb{Z}_{2} * \mathbb{Z}_{2}$ as well as in the group of half integers $\mathbb{Z} / 2$.

Similarly, $\mathbb{F}_{2}$ may be embedded in $\mathbb{Z}_{2} * \mathbb{Z}_{2} * \mathbb{Z}_{2}$ and in $\mathbb{Z} / 2 * \mathbb{Z}_{2}, \mathbb{F}_{3}$ in $\mathbb{Z}_{2} * \mathbb{Z}_{2} * \mathbb{Z}_{2} * \mathbb{Z}_{2}$ and in $\mathbb{Z} / 2 * \mathbb{Z}_{2} * \mathbb{Z}_{2}$ and $\mathbb{Z} / 2 * \mathbb{Z} / 2$, and so on.

4.3. It is intriguing that the numbers emerging from this calculation on free groups should be the remainder terms in a Laurent series. Is there some way to carry out this polar decomposition so that these appear more naturally? Does the angle $\arctan (1 / \sqrt{k})$ have a geometric interpretation?

4.4. The polar decomposition of $1+e^{i \theta}(-\pi<\theta \leqslant \pi)$ implicitly involves topological surgery in a very elementary form. The function $e^{i \theta} \mapsto 1+e^{i \theta}$ is continuous on the circle, as is its positive part $e^{i \theta} \mapsto 2 \cos \theta / 2$. However the polar part, $e^{i \theta} \mapsto e^{(i \theta) / 2}$ is not continuous at $e^{i \pi}=-1$ and so we cut the circle at this point, figure 3 . In doing so, we move outside $C(\mathbb{T})$ into $L^{\infty}(\mathbb{T})$ or, equivalently, from $C_{\tau}^{*}(\mathbb{Z})$ into $V N(\mathbb{T})$, but not all of $L^{\infty}(\mathbb{T})$ is required for the polar decomposition. The parametrisation $\theta \mapsto e^{i \theta}$ : $[-\pi, \pi] \rightarrow \mathbb{T}$ induces an embedding $C(\mathbb{T}) \rightarrow C([-\pi, \pi])$ and it is in $C([-\pi, \pi])$ that the polar decomposition takes place.
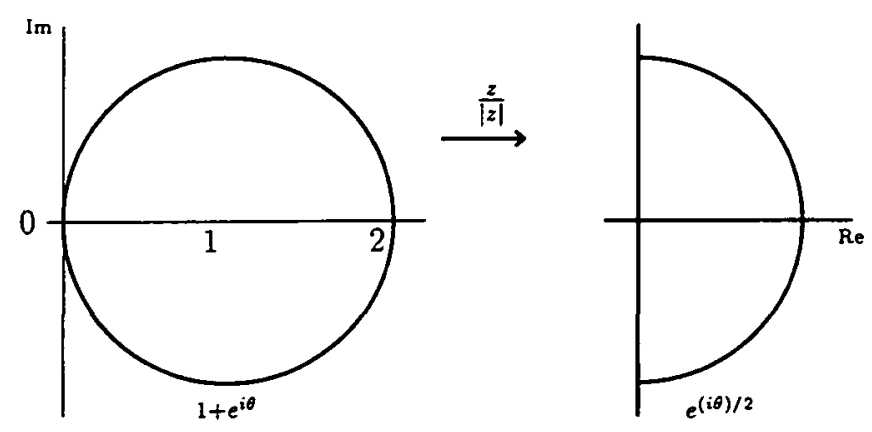

Figure 3. 
Now thinking of $C_{\tau}^{*}\left(\mathbb{F}_{k}\right)$ as an algebra of 'continuous functions' on a noncommutative 'space', $T=\lambda(e)+\sum_{j=1}^{k} \lambda\left(x_{j}\right)$ and $P$ belong to $C_{\tau}^{*}\left(\mathbb{F}_{k}\right)$ but the polar part, $U$, belongs only to $V N\left(\mathbb{F}_{k}\right)$ and so is not 'continuous'. Analogy suggests that the polar decomposition be regarded as 'cutting' the underlying or supporting 'space' of $C_{r}^{*}\left(\mathbb{F}_{k}\right)$. The closed form of the polar decomposition computed here suggests that it is a particularly natural way to 'cut' this 'space'. The $C^{*}$-algebra generated by $U$ and $C_{r}^{*}\left(\mathbb{F}_{k}\right)$, to be denoted by $\mathfrak{A}_{k}$, is the smallest algebra in which the polar decomposition may be formed. Continuing the analogy, the supporting 'space' of $\mathfrak{A}_{k}$ is the result of 'cutting' the 'space' supporting $C_{\tau}^{*}\left(\mathbb{F}_{k}\right)$ and the embedding $C_{\tau}^{*}\left(\mathbb{F}_{k}\right) \rightarrow \mathfrak{A}_{k}$ corresponds to a 'continuous function' generalising the parametrisation of $\mathbb{T}$ by $[-\pi, \pi]$.

The parametrisation $\theta \mapsto e^{i \theta}$ identifies the endpoints of the interval $[-\pi, \pi]$ and thus is the reverse of cutting the circle at $e^{i \theta}=-1$. A similar understanding of noncommutative surgery might possibly be obtained by describing how irreducible representations of $\mathfrak{A}_{k}$ relate to those of $C_{r}^{*}\left(\mathbb{F}_{k}\right)$ under the embedding $C_{r}^{*}\left(\mathbb{F}_{k}\right) \rightarrow \mathfrak{A}_{k}$. There is a natural class of representations of $C_{r}^{*}\left(\mathbb{F}_{k}\right)$ to be considered, namely the restrictions to $C_{\tau}^{*}\left(\mathbb{F}_{k}\right)$ of the irreducible representations of $C_{r}^{*}\left(\mathbb{G}_{k}\right)$ arising in the direct integral decomposition of the regular representation of $\mathbb{G}_{k}$ over the spectrum of the operator $A$ in $C_{r}^{*}\left(\mathbb{G}_{k}\right)$, see [5].

It is to be expected that the 'space' supporting $\mathfrak{A}_{k}$ is connected in the sense that $K_{0}\left(\mathfrak{A}_{k}\right)$ is trivial, see [4]. However the polar decomposition of $\delta_{0}+\delta_{n}$ in $C_{\tau}^{*}(\mathbb{Z})$ cuts the circle into $n$ pieces and similar can be expected of subgroups having finite index in $\mathbb{F}_{k}$. More precisely, each subgroup with finite index in $\mathbb{F}_{k}$ is isomorphic to a free group with a finite number of generators, $y_{1}, \ldots, y_{m}$ say. Let $\mathfrak{A}$ denote the $C^{*}$-algebra obtained by adjoining to $C_{r}^{*}\left(\mathbb{F}_{k}\right)$ the unitary from the polar decomposition of $\lambda(e)+\sum_{j=1}^{k} \lambda\left(y_{j}\right)$. Does $K_{0}(\mathfrak{A})$ depend only on the index of the subgroup? We have seen here that at least one idempotent arises when the subgroup has index two.

Similarly, algebras obtained by adjoining unitaries from several polar decompositions can be expected to have non-trivial $K$-groups. Can $C_{r}^{*}\left(\mathbb{F}_{k}\right)$ be analysed in terms of its pieces as it is 'cut' by successive polar decompositions and can it be reconstructed from these pieces?

When the concept of continuous function is first defined in elementary calculus it is usual to illustrate it by giving examples of functions which are not continuous. Indeed, the intuitive notion of a continuous function is that of one without breaks or jumps, see [1, Section 3.4, p. 91], and in some languages that is the literal meaning of 'continuous'. It is natural then to investigate $C_{r}^{*}\left(\mathbb{F}_{k}\right)$, or any $C^{*}$-algebra, and to attempt to make some sense of the notion that its elements are 'continuous' by studying associated discontinuous elements. 
4.5. It is in general difficult to determine whether or not functions in $\ell^{2}\left(\mathbb{F}_{k}\right)$ belong to $V N\left(\mathbb{F}_{k}\right)$ or $C_{r}^{*}\left(\mathbb{F}_{k}\right)$ and to compute operator norms. This is usually done by considering smooth elements, which means imposing a growth condition on the function which forces it to belong to $l^{1}\left(\mathbb{F}_{k}\right)$, or by appealing to some result such as Haagerup's inequality [6]. The cause of this difficulty is not noncommutativity because it is even difficult to decide whether a given function in $\ell^{2}(\mathbb{Z})$ is the Fourier transform of a continuous or bounded function on $\mathbb{T}$, [11, 4.3, p. 78].

In the commutative case however there is an extra technique for writing down measurable or continuous functions. Referring once more to elementary calculus, the examples of continuous functions presented are all smooth, as in the noncommutative case, or piecewise smooth. Piecewise constant (or simple) functions and piecewise linear functions belong to $L^{\infty}(\mathbb{T})$. If the values of a piecewise linear function are matched up correctly, then it will belong to $C(\mathbb{T})$. The freedom to write down piecewise linear functions is very useful, see for example the proof that $C(\mathbb{T})$ has Schauder basis given in [10, Theorem IIID.25].

Linear combinations of idempotents and partial isometries would seem to be the natural analogue of piecewise constant functions in $V N\left(\mathbb{F}_{k}\right)$. Is there a natural analogue of piecewise linear functions in $V N\left(\mathbb{F}_{k}\right)$ and $C_{r}^{*}\left(\mathbb{F}_{k}\right)$ ? A continuous function on $\mathbb{T}$ can be approximated uniformly by functions constant or linear on the intervals $((2 \pi j) / n,(2 \pi(j+1)) / n], j=0,1, \ldots, n-1$. Can an arbitrary operator in $C_{r}^{*}\left(\mathbb{F}_{k}\right)$ be approximated in norm by linear combinations of idempotents and partial isometries in $V N\left(\mathbb{F}_{k}\right)$, where the idempotents and partial isometries arise from the polar decomposition of $\lambda(e)+\sum_{j=1}^{m} \lambda\left(y_{j}\right)$ with $\left\langle y_{1}, y_{2}, \ldots, y_{m}\right\rangle$ having finite index in $\mathbb{F}_{k}$ ? It seems likely that such as approximation would rely on the residual finiteness of $\mathbb{F}_{k}$.

\section{REFERENCES}

[1] D. Bressoud, A radical approach to real analysis (Mathematical Association of America, Washington, 1994).

[2] P. Cartier, 'Harmonic analysis on trees', Proc. Symp. Pure Math. 26 (1973), 419-424.

[3] A. Connes, Noncommutative geometry (Academic Press, Paris, 1994).

[4] E.G. Effros, 'Why is the circle connected: an introduction to quantized topology.', Math. Intelligencer 11 (1989), 27-34.

[5] A. Figà-Talamanca and M. A. Picardello, Harmonic analysis on free groups (Marcel Dekker, Inc., New York, 1983).

[6] U. Haagerup, 'An example of a non nuclear $C^{*}$-algebra which has the metric approximation property', Invent. Math. 50 (1979), 279-293.

[7] P. Halmos, A Hilbert space problem book (Spinger-Verlag, Berlin, Heidelberg, New York, 1982).

[8] T. Pytlik, 'Radial functions on free groups and a decomposition of the regular representation into irreducible components', J. Reine Angew. Math. 326 (1981), 124-135. 
[9] S. Sawyer, 'Isotropic random walks in a tree', Z. Wahrsch. Verw. Gebiete 42 (1978), 279-292.

[10] P. Wojtaszcyk, Banach spaces for analysts (Cambridge University Press, Cambridge, 1991).

[11] A. Zygmund, Trigonometrical series (Dover Publaications, New York, 1955).

Department of Mathematics

University of Newcastle

Callaghan NSW 2308

Australia

e-mail: george@math.newcastle.edu.au 